Check for updates

Cite this: RSC Adv., 2018, 8, 20440

\title{
Aryl fluoride functionalized graphene oxides for excellent room temperature ammonia sensitivity/ selectivity $\dagger$
}

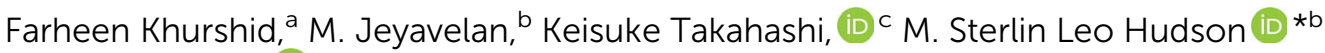 \\ and S. Nagarajan $(\mathbb{D} * a$
}

\begin{abstract}
Herein, we report the covalent functionalization of graphene oxide (GO) through "click" reaction and its applications towards ammonia sensing. This inimitable method of covalent functionalization involves linking GO with azide moiety and click coupling of different derivatives of aryl propargyl ether, which enhances the sensitivity towards ammonia. The functionalized GO were characterized using NMR, XRD, SEM, FT-IR, Raman, UV-Vis, TGA and DSC. Compared to pristine GO, the GO functionalized with Ar samples (GO-Ar) exhibit excellent room temperature ammonia sensing properties with good response/ recovery characteristics. It has been observed that 2,3-difluoro and 2,3,4-trifluoro substituted aryl propargyl ether functionalized $\mathrm{GO}$ (GO-Ar2 and GO-Ar3) shows superior ammonia sensing with response/recovery of $63 \% / \sim 90 \%$ and $60 \% / 100 \%$, respectively at $20 \mathrm{ppm}$. The GO-Ar3 exhibits high sensitivity towards ammonia at 20-100 ppm. Computational studies supports the high sensitivity of GO-

Ar towards ammonia due to its high adsorption energy.
\end{abstract}

Received 1st March 2018

Accepted 29th May 2018

DOI: $10.1039 / c 8 r a 01818 a$

rsc.li/rsc-advances

\section{Introduction}

Materials suitable for gas detection have recently gained popularity due to their potential application towards detection of toxic and flammable gases such as $\mathrm{H}_{2}, \mathrm{CH}_{4}, \mathrm{CO}, \mathrm{NO}_{x}$, and $\mathrm{NH}_{3}$. Gas sensors are widely used in many commercial and industrial applications such as exhaust gas detection as well as flammable and explosive gas detection. ${ }^{1}$ Among all toxic gases, ammonia $\left(\mathrm{NH}_{3}\right)$, a colourless and highly toxic gas, draws particular interest. While blood ammonium concentration is normally $<50 \mu \mathrm{mol} \mathrm{L}^{-1}$, an increase to nearly $200 \mu \mathrm{mol} \mathrm{L}^{-1}$ can lead towards convulsions or a comatose state. Exposure of $>$ $55 \mathrm{ppm}$ concentration of ammonia in air for 48 hours can also cause respiratory and cardiovascular diseases. ${ }^{2-4}$ Hence, the development and optimization of low concentration $\mathrm{NH}_{3}$ sensing material is very important. Graphene is found to be a promising material for gas sensing applications because of its large surface area $\left(2630 \mathrm{~m}^{2} \mathrm{~g}^{-1}\right.$ theoretical). ${ }^{5}$ The $2 \mathrm{D}$ crystalline honeycomb framework of carbon atoms on a graphene surface can provide a large sensing area per unit volume to adsorbed

${ }^{a}$ Department of Chemistry, Central University of Tamil Nadu, Thiruvarur - 610005, India. E-mail: snagarajan@cutn.ac.in; Tel: +91-9489051816

${ }^{b}$ Department of Physics, Central University of Tamil Nadu, Thiruvarur-610005, India. E-mail: msterlinleo@cutn.ac.in; Tel: +91-9486860214

${ }^{\circ}$ Center for Materials Research By Information Integration $\left(\mathrm{CMI}^{2}\right)$, National Institute for Materials Science (NIMS), 1-2-1 Sengen, Tsukuba, Ibaraki 305-0047, Japan

$\uparrow$ Electronic supplementary information (ESI) available. See DOI: 10.1039/c8ra01818a gas molecules. ${ }^{6}$ The electrostatic interaction between graphene and adsorbates could vary from weak van der Waals interaction to strong covalent interaction. These interactions may lead towards a change in the electronic system of graphene. ${ }^{7}$ Gas sensing properties of graphene and sensitivity of graphene has been investigated by several researchers. ${ }^{8}$ However, the chemical inertness of a pure graphene surface reduces its ammonia sensing performance. Graphene-based sensors for detecting low concentration ammonia gas become a prime area of sensor research..$^{9-11}$ It has been observed that graphene functionalized with high electronegative elements such as fluorine or boron enhances its sensitivity towards ammonia gas. ${ }^{12}$ Katkov et al. reported that experimental observations of about $10.2 \%$ increase in ammonia sensitivity (at $10000 \mathrm{ppm}$ ) of graphene after functionalization with fluorine. ${ }^{13}$ Additionally, Ruitao Lv et al. ${ }^{14}$ found that upon boron doping, the sensitivity of graphene towards ammonia has increased nearly 100 times. $^{13}$ Nowadays, due to limitations of large-scale synthesis of single layer graphene, significant research is focused on oxides of graphite for detection of gases such as ammonia, $\mathrm{NO}_{2}$, humidity, and air flow. ${ }^{12,15-20}$ In graphite oxide (GO), the $\mathrm{sp}^{2}$ hybridized carbon surface of graphite get modified by oxygen functional groups like carboxylic, epoxy, and ether groups. Hence, the GO surface becomes a $\mathrm{sp}^{3}$ hybridized surface, resulting in more active sites for gaseous species (adsorbates). ${ }^{21}$ Furthermore, the high concentration of oxygen-containing functional groups in $\mathrm{GO}^{22}$ offers a high degree of selectivity for the detection of toxic gases at room temperature. ${ }^{23}$ Bannov et al. observed that GO based sensors have an ammonia 
sensitivity of $7.4 \%$ (at $500 \mathrm{ppm}$ ) at room temperature. ${ }^{23} \mathrm{Wu}$ et al. observed graphene oxide/polyaniline nanocomposite has an ammonia sensitivity of $11.33 \%$ (at 100 ppm) ammonia sensitivity. ${ }^{24}$ Reports suggested that an enhancement in selectivity and sensitivity response of graphene towards ammonia gas detection when it is functionalized/decorated with conducting polymer, ${ }^{25}$ metal oxides, ${ }^{26}$ and fluorine substitution ${ }^{27}$ via covalent and non-covalent functionalization. Many commercial ammonia gas sensors have high sensitivity at high temperature and poor sensitivity at low temperature. More recently, research is being focused on developing gas sensors with high sensitivity at low temperature and near room temperature. Therefore, the functionalization of GO with terminal azide moiety and different derivatives of aryl propargyl ether through $\mathrm{Cu}$ catalyzed Huisgen 1,3-dipolar cycloaddition (click reaction) is investigated. The electron withdrawing group functionalized aryl propargyl ethers are chosen to understand its gas role on the GO surface.

\section{Experimental section}

\subsection{Materials}

Graphite powder (300 mesh) $N$-(3-dimethylaminopropyl)- $N^{\prime}$ ethylcarbodiimide hydrochloride (EDC $\mathrm{HCl}$, 99\%), propargyl bromide, chloropropylamine, sodium azide, $\mathrm{CuBr}$ and $\mathrm{N}$ hydroxysuccinimide were purchased from Sigma Aldrich. $N, N, N^{\prime}, N^{\prime \prime}, N^{\prime \prime}$-pentamethyldiethylenetriamine (PMDETA) was purchased from Alfa Aesar.

\subsection{Synthesis of graphene oxide (GO)}

Graphene Oxide (GO) was synthesized by using modified Hummers method. ${ }^{28}$ About $5 \mathrm{~g}$ of graphite powder and $2.5 \mathrm{~g}$ of $\mathrm{NaNO}_{3}$ were mixed together with concentrated $\mathrm{H}_{2} \mathrm{SO}_{4}(108 \mathrm{ml})$ under constant stirring. Then $12 \mathrm{ml}$ of $\mathrm{H}_{3} \mathrm{PO}_{4}$ was added to the $2: 1$ graphite- $\mathrm{NaNO}_{3}$ mixture kept under ice cooled bath and continued stirring for $10 \mathrm{~min}$. Afterwards, $15 \mathrm{~g}$ of $\mathrm{KMnO}_{4}$ was gradually added to the mixture with constant stirring. The suspension was continuously stirred for 2 hours under ice cold conditions and continued stirring at $40{ }^{\circ} \mathrm{C}$ for 1 hour. The reaction mixture was diluted with deionized water at $98^{\circ} \mathrm{C}$. The resultant final product was dried overnight at $60{ }^{\circ} \mathrm{C}$ and then subjected for characterization.

\subsection{Synthesis of azide functionalized GO}

3-Azidoropan-1-amine (7.5 mmol) was condensed with GO (1 g) under catalytic action of EDC $(2.5 \mathrm{mmol})$ and NHS $(2.2 \mathrm{mmol})$. The reaction mixture was stirred at room temperature for 24 hours, and upon completion the reaction mixture was centrifuged at $1000 \mathrm{rpm}$ for $5 \mathrm{~min}$. The suspension was collected and washed several times with deionized water to get GO-N $\mathrm{N}_{3}$.

\subsection{Click reaction of GO- $\mathrm{N}_{3}$ to aryl propargyl ether}

The functionalization of GO with different derivatives of aryl propargyl ether was done by click reaction. ${ }^{29}$ Aryl propargyl ether $(0.1 \mathrm{ml})$ was added to a round bottom flask containing GO$\mathrm{N}_{3}\left(0.016 \mathrm{ml}, 50 \mathrm{mg} \mathrm{ml}^{-1}\right.$ of water). Then DMF $(1 \mathrm{ml})$, CuBr $(0.973 \mathrm{mmol})$, and PMDETA $(0.293 \mu \mathrm{l})$ were added to the flask under nitrogen atmosphere and stirred for 3 hours at $60{ }^{\circ} \mathrm{C}$. The reaction product was centrifuged at $500 \mathrm{rpm}$ for $10 \mathrm{~min}$. The residue was washed five times with DMF, and dried overnight under vacuum at $60{ }^{\circ} \mathrm{C}$. The samples were collected and stored in vacuum desiccator. The evidence for the functionalization of Ar moiety on terminal azide GO $\left(\mathrm{GO}-\mathrm{N}_{3}\right)$ discussed in the ESI (Fig. S5†).

\subsection{Scheme for the synthesis of sensing molecules}

As from Fig. 1 (scheme), the derivatives aryl propargyl ether were synthesized by a reaction between propargyl bromide and respective phenols (2,4,6-trimethyl (Ar1), 2,3-difluro (Ar2) and 2,3,4-trifluro (Ar3)) in the presence of $\mathrm{K}_{2} \mathrm{CO}_{3}$ as reported earlier. ${ }^{33}$ The functionalized GO (GO-Ar) samples were prepared by a Husigen's 1,3-dipolar cycloaddition reaction between aryl propargyl ether and azido-terminated GO (GO-N $)_{3}$ utilizing

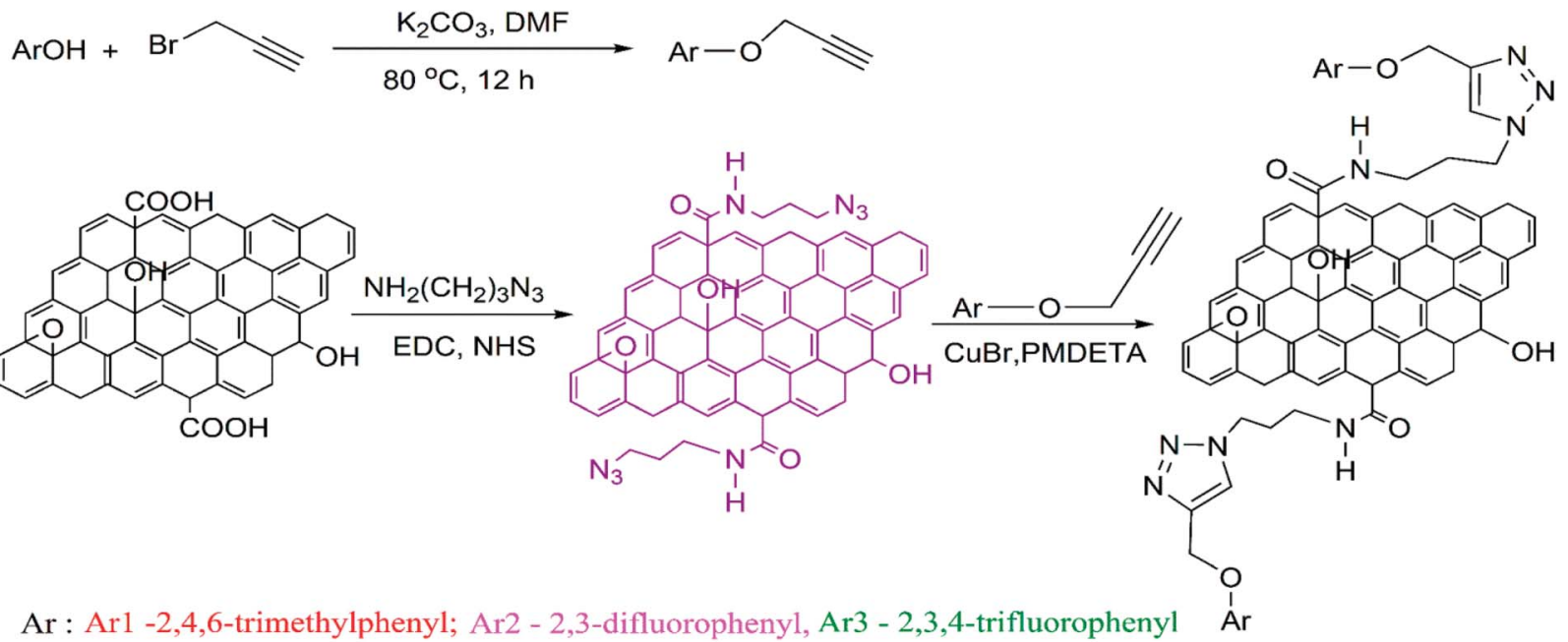

Fig. 1 Scheme: synthesis of aryl propargyl ethers and functionalization of GO-N3 with derivatives of aryl propargyl ethers through click reaction. 
$\mathrm{CuBr}$ as catalyst. After 5 minutes, $15 \mathrm{ml}$ of $\mathrm{H}_{2} \mathrm{O}_{2}$ was added into the suspension. The reaction product was filtered with $\mathrm{G} 4$ sintered crucible and repeatedly washed with deionized water and $5 \% \mathrm{HCl}$ solution.

\subsection{Structural, microstructural, thermal and spectral analysis}

${ }^{13} \mathrm{C}$ and ${ }^{1} \mathrm{H}$ NMR spectra were recorded using a Bruker $400 \mathrm{MHz}$ spectrophotometer. Chemical shift values $(\delta)$ are reported in ppm and calibrated with the residual solvent peak $\mathrm{CDCl}_{3} \delta=$ $7.2600 \mathrm{ppm}$ for ${ }^{1} \mathrm{H}, \delta=77.16$ for ${ }^{13} \mathrm{C}$ and tetramethylsilane $(\delta=$ $0.00)$. The structural analysis of samples was carried out using a powder X-ray diffractometer (PANalytical X'Pert PRO) equipped with $\mathrm{X}^{\prime}$ Celerator position sensitive detector using $\mathrm{Cu} \mathrm{K}_{\alpha}$ radiation of wavelength $\lambda=1.5401 \AA$. Microstructure analysis of the samples were characterized by using JEOL scanning electron microscope (SEM). Fourier transform infrared (FT-IR) spectra and Raman spectra were obtained using Agilent-Cary 630 and EZRaman-N-785 spectrometer, respectively. Differential scanning calorimetry (DSC) and thermogravimetric analysis (TGA) of the samples were recorded between $40^{\circ} \mathrm{C}$ and $400{ }^{\circ} \mathrm{C}$ at the dynamical heating rate of $10{ }^{\circ} \mathrm{C} \min ^{-1}$ under constant nitrogen flow ( $20 \mathrm{ml} \mathrm{min}^{-1}$ ) using a PerkinElmer STA 8000. The BET surface area of the samples were calculated by using Autosorb iQ under nitrogen atmosphere at $77 \mathrm{~K}$.

\subsection{Sensing device fabrication and measurements}

The sensor electrode was fabricated by a gold film coating using thermal evaporation technique on a patterned glass substrate. The gold coated electrode was with a dimension of $12 \mathrm{~mm}(L) \times$ $8 \mathrm{~mm}(W) \times 200 \mathrm{~nm}(t)$ with $1 \mathrm{~mm}$ strips spacing. The GO based sensing film was prepared from $50 \mathrm{mg}$ sample dispersed in $2 \mathrm{ml}$ of ethanol and deposited on the gold coated electrode by using drop-casting method. Afterwards, the sensor was dried at $45{ }^{\circ} \mathrm{C}$ for 1 hour. The sensing measurements were carried out using BioLogic VSP-300 Impedance analyzer at an input supply of $1 \mathrm{~V}$ $(f=1 \mathrm{kHz})$. The ammonia solutions of varying volumes (corresponding to the vapour concentrations ranging from $20 \mathrm{ppm}$ to $100 \mathrm{ppm}$ ) were dropped using a micropipette into a sealed glass chamber of volume 1.77 litre. Fig. 2(a, b and c) represents the schematic $\mathrm{NH}_{3}$ sensing setup, used for monitoring the sensor response by switching between $\mathrm{NH}_{3}$ atmosphere and ambient air. The sensing measurements were continuously recorded by varying the $\mathrm{NH}_{3}$ concentrations from $20 \mathrm{ppm}$ to $100 \mathrm{ppm}$ at an interval of $5 \mathrm{~min} \mathrm{ON} / \mathrm{OFF}$ duration. The concentration of injected analyte $\left(\mathrm{NH}_{3}\right)$ in the chamber was calculated in $\mathrm{ppm}$ according to the following equation: ${ }^{30,31}$

$$
C=\frac{22.4 \rho T V_{\mathrm{S}}}{273 M V} \times 10^{3}
$$

where, $C$ is the concentration of ammonia vapour inside the air sealed chamber in ppm, $\rho$ is the density of liquid ammonia solution $\left(\mathrm{g} \mathrm{ml}^{-1}\right), T$ is the testing temperature $(\mathrm{K}), V_{\mathrm{S}}$ is the volume of ammonia solution $(\mu \mathrm{l}), M$ is the molecular weight of ammonia $\left(\mathrm{g} \mathrm{mol}^{-1}\right)$ and $V$ is the volume of the chamber (L). After ensuring a steady current output from the sensor in open

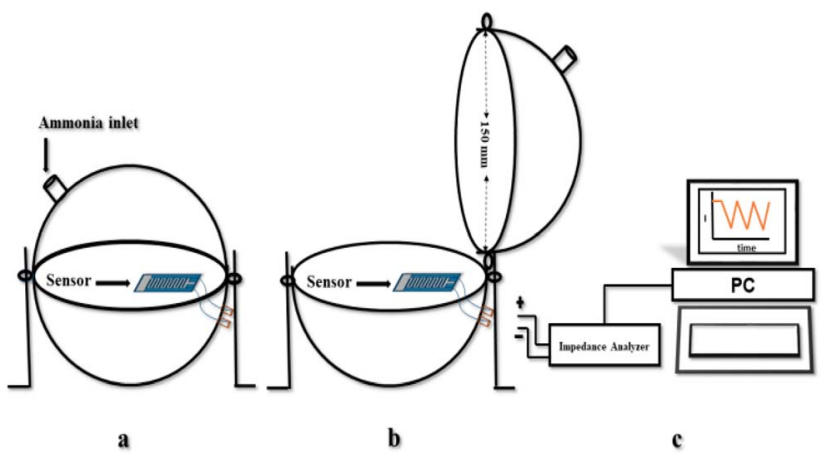

Fig. 2 Schematic gas sensing setup: (a) sensor inside a sealed chamber under ammonia atmosphere, (b) sensor in ambient air, and (c) impedance measurement system.

atmosphere for about 100 seconds, $\mathrm{NH}_{3} \mathrm{ON} / \mathrm{OFF}$ cycles has been initiated. The change in sensor resistance was monitored at $28{ }^{\circ} \mathrm{C}$ by switching the sensor response between $\mathrm{NH}_{3}$ atmosphere and ambient air. ${ }^{32}$ Ammonia sensing under relative humidity $(\mathrm{RH})$ conditions were carried out by using saturated salt solutions of $\mathrm{LiCl}, \mathrm{MgNO}_{3}$ and $\mathrm{K}_{2} \mathrm{SO}_{4}$ to obtain the $\mathrm{RH}$ values of $11 \%, 51 \%$ and $97 \%$, respectively. The $\mathrm{RH}$ inside the chamber was measured by using digital hygrometer. Once the RH was achieved, the response measurements were determined after the sensor is exposed to $20 \mathrm{ppm}$ of ammonia for $10 \mathrm{~min}$ duration. ${ }^{32}$

\section{Results and discussion}

\subsection{Structural, microstructural, thermal and surface area analysis}

Spectral studies confirmed the formation of the functionalized GO described in the Scheme shown in Fig. 1. The detailed NMR structural analysis of aryl propargyl ethers (Ar1, Ar2 and Ar3) are given in ESI (Fig. S1(a) to S3(b)†). The Fig. 3(a) displays the XRD pattern of GO and functionalized GO. The strong diffraction peak of GO observed at $2 \theta=11.5^{\circ}$ corresponding to (001) plane having $d$-spacing of $0.75 \mathrm{~nm}$ confirms the oxidation of graphite. ${ }^{28}$ After the functionalization of GO with azide moiety $\left(\mathrm{N}_{3}\right)$, the diffraction peak of GO has shifted to low diffraction angle at $2 \theta=9.5^{\circ}$ corresponding to $d$-spacing of $0.92 \mathrm{~nm}$. The attachment of azide moiety $\left(\mathrm{N}_{3}\right)$ on GO surface leads to an increased $d$-spacing of GO- $\mathrm{N}_{3}$.

Whereas, an additional diffraction peak observed at $2 \theta=$ $19.4^{\circ}$ for $\mathrm{GO}-\mathrm{N}_{3}$ might be due to the partial reduction of GO during the reaction process. ${ }^{29}$ In addition, after functionalization of GO- $\mathrm{N}_{3}$ with Ar1, Ar2 and Ar3, it was observed that (001) peak becomes broader and further shifted towards lower angle than GO-N $\mathrm{N}_{3}$ corresponding to the $d$-spacing's of $2.43 \mathrm{~nm}$, $2.20 \mathrm{~nm}$ and $2.55 \mathrm{~nm}$, respectively. Whereas the partial reduction peaks are observed in GO-Ar1, GO-Ar2 and GO-Ar3 at $23.4^{\circ}$, $24.5^{\circ}$ and $20.2^{\circ}$. This suggest that the functionalization causes vacancies in GO. ${ }^{34}$ These vacancies also act as an active site for the ammonia adsorption. 

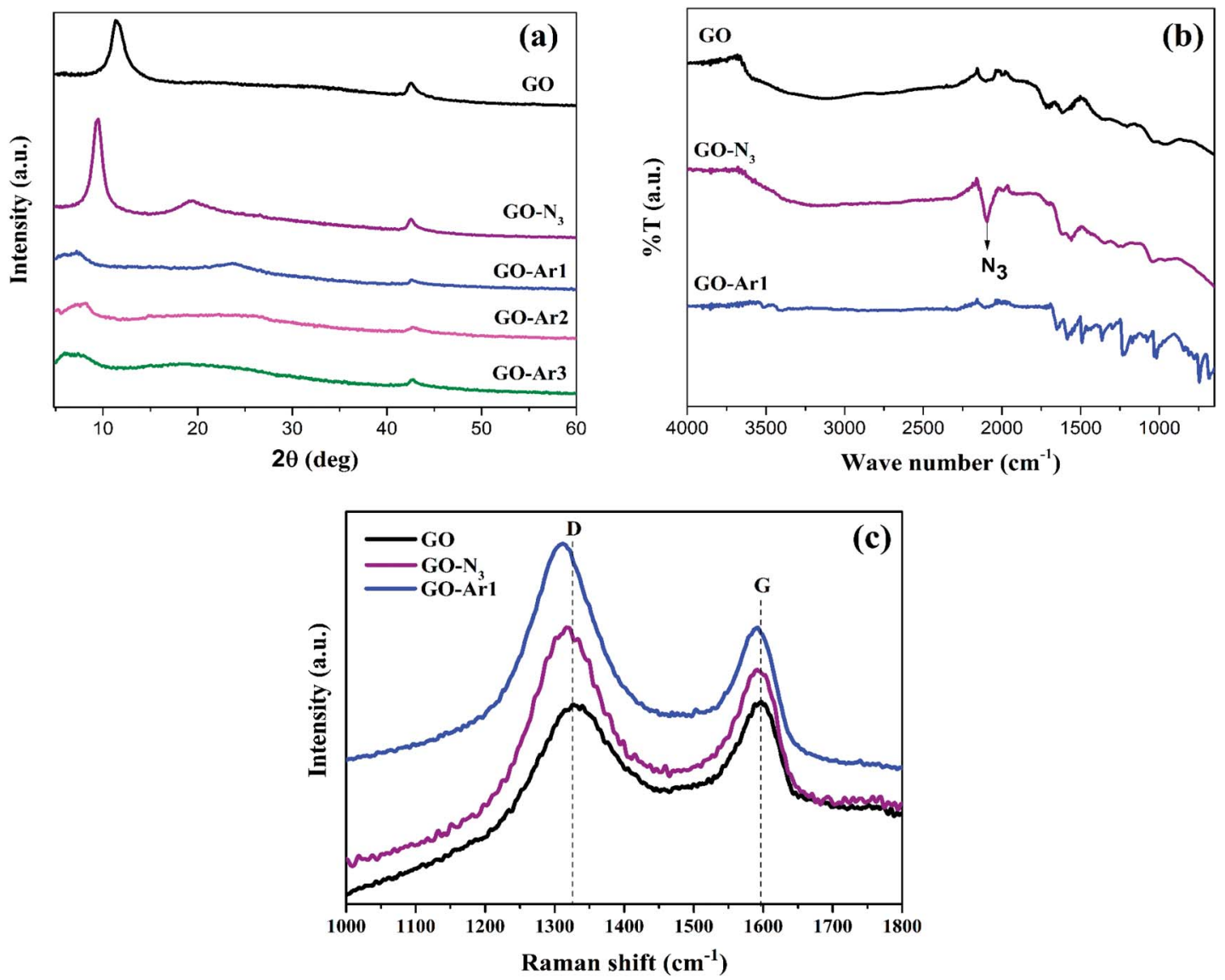

Fig. 3 (a) XRD Spectra of GO and Functionalized GO, (b) and (c) FT-IR and Raman spectra of GO, GO-N3 and GO-Ar1.

Fig. 3(b) portrays FT-IR spectra of GO, GO-N 3 and GO-Ar1 and the peaks found in $\mathrm{GO}$ at 1104,1720 , and $3282 \mathrm{~cm}^{-1}$ corresponding to epoxy, carboxylic $(\mathrm{COOH})$, and hydroxyl $(\mathrm{OH})$ groups, respectively confirms the oxygen functionalities on GO surfaces. ${ }^{29}$ whereas, in GO- $\mathrm{N}_{3}$ a sharp absorption peak has been observed at $2106 \mathrm{~cm}^{-1}$, assigned to azide moiety $\left(\mathrm{N}_{3}\right)$. This result suggests the successful condensation reaction between GO and azide moiety. ${ }^{35}$ Unlike GO and GO-N $\mathrm{N}_{3}$, the GO-Ar shows several additional spectral peaks at $1044 \mathrm{~cm}^{-1}(\mathrm{C}-\mathrm{N}), 1142 \mathrm{~cm}^{-1}$ (ester linkage), $1578 \mathrm{~cm}^{-1}(\mathrm{C}=\mathrm{N}), 1640 \mathrm{~cm}^{-1}(\mathrm{~N}-\mathrm{H})$, and $1680 \mathrm{~cm}^{-1}(\mathrm{C}=\mathrm{O})$, corresponding to coupling of aryl propargyl ether with terminal azide moiety on GO were observed. The Raman spectral analysis was carried out to understand the interaction between $\mathrm{GO}$ and $\mathrm{N}_{3} / \mathrm{Ar}$ moieties. The representative Raman spectra of GO, GO-N 3 and GO-Ar1 are shown in Fig. 3(c). It has been observed two prominent peaks; the $\mathrm{G}$ band spectrum observed at $1594 \mathrm{~cm}^{-1}$, corresponding to the doubly degenerate zone-center $\mathrm{E}_{2 \mathrm{~g}}$ mode and a broad $\mathrm{D}$ band spectrum at $1330 \mathrm{~cm}^{-1}$ due to the formation of $\mathrm{sp}^{3}$ hybridized carbon bonds during the oxidation of $\mathrm{GO}^{34}$ The intensity ratio of $\mathrm{D}$ and G bands $\left(I_{\mathrm{D}} / I_{\mathrm{G}}\right)$ of Raman spectra is generally used to characterize the level of defect density in graphene. ${ }^{36}$ From Fig. 3(c), it has been observed that the ratio of $I_{\mathrm{D}} / I_{\mathrm{G}}$ increases from GO $(0.995)>$ GO$^{-} \mathrm{N}_{3}$ (1.102)>GO-Ar2 (1.376), which reveals the increased defect density in graphene due to the functionalization of $\mathrm{N}_{3}$ and $\mathrm{Ar}$ moieties at the edges of GO.

The Fig. 4 displays, the morphologies of pristine GO-Ar1 and GO-Ar3. The SEM images shows the surface texture of the samples. A similar morphologies were observed for Ar1 functionalized GO and Ar3 functionalized GO.

Fig. 5 displays the TGA curves of GO, GO- $\mathrm{N}_{3}$ and GO-Ar samples. The thermal decomposition measurements were done between $40{ }^{\circ} \mathrm{C}$ to $400{ }^{\circ} \mathrm{C}$ under dynamic heating rate of $10{ }^{\circ} \mathrm{C} \min ^{-1}$ it has been observed from the TGA curve of GO,
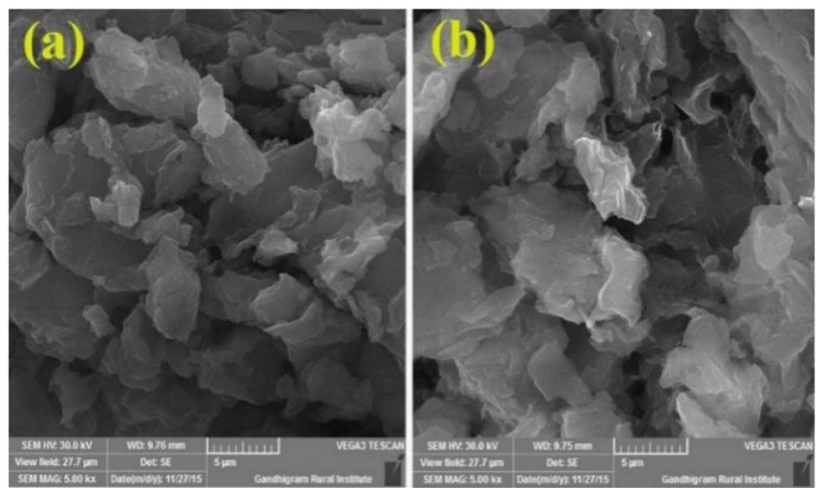

Fig. 4 SEM images (a) GO-Ar1 and (b) GO-Ar3 under the magnification of $5 \mathrm{kx}$. 


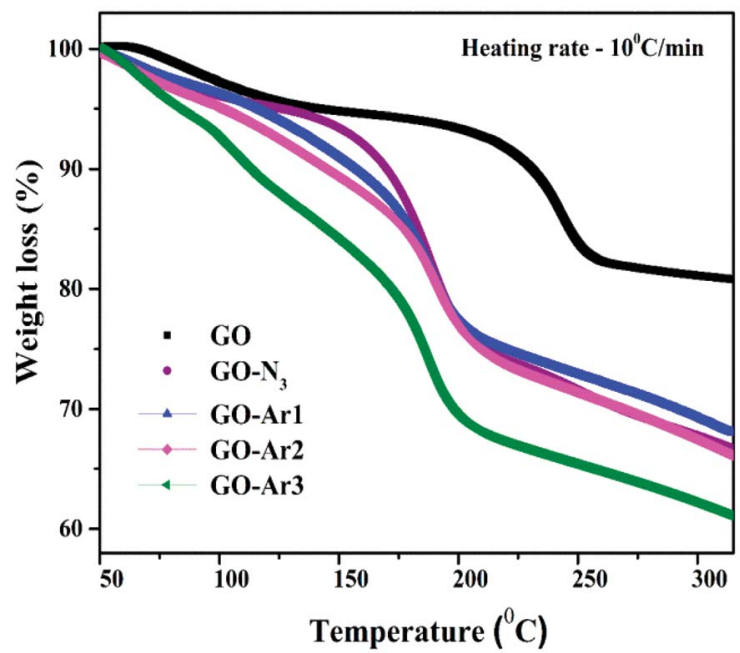

Fig. 5 TGA curves of the GO and Functionalized GO samples.

a major weight loss $(\sim 12 \%)$ occurred between $100{ }^{\circ} \mathrm{C}$ to $250{ }^{\circ} \mathrm{C}$ indicates the removal of volatiles and pyrolysis of oxygen-related functional groups on GO surfaces..$^{37,38}$ In GO the overall weight loss of $20 \%$ has been observed from 50 to $250{ }^{\circ} \mathrm{C}$ due to the decomposition of oxygen and other functional groups. Whereas the major decomposition temperature of GO $\left(220{ }^{\circ} \mathrm{C}\right)$ has shifted towards low temperature for all functionalized GO samples due to the substitution of $\mathrm{N}_{3}, \operatorname{Ar} 1, \operatorname{Ar} 2$ and Ar3. In addition to that, the Azide and the aryl propargyl ether functionalized GO samples exhibit higher weight losses (28, 27, 29 and 35\%)from 50 to $250{ }^{\circ} \mathrm{C}$ than GO, due to the decomposition of higher molecular weight aryl propargyl ether derivatives. It has been observed that, the functionalized GO samples have lower decomposition temperatures $\left(\sim 179,171,167\right.$ and $\left.160^{\circ} \mathrm{C}\right)$ than pristine GO $\left(235^{\circ} \mathrm{C}\right)$.

The BET surface area of the samples was analysed from $\mathrm{N}_{2}$ adsorption/desorption isotherm at $77 \mathrm{~K}$ as shown in Fig. 6 and the corresponding isotherms and multipoint BET plot (insert graph in Fig. 6) reveals that the GO, GO-Ar2 and GO-Ar3 samples have surface area of 9.34, 11.445 and $12.245 \mathrm{~m}^{2} \mathrm{~g}^{-1}$ respectively.

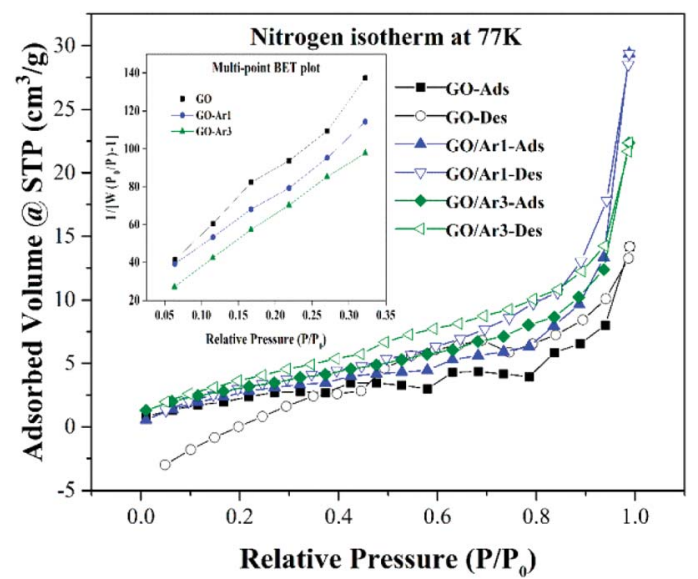

Fig. 6 BET curves and the insert graph represents the multi-point plot of GO, GO-Ar1 and GO-Ar3.
As inferred from BET measurement the GO samples has very low surface area. However, it slightly increases when increasing the order of functionalization at edges of GO. It has been observed from that, the isotherm of all the samples resemble with the type IV (IUPAC) isotherm and it signifies that the materials are mesoporous in nature.

\subsection{Ammonia sensing analysis}

The ammonia $\left(\mathrm{NH}_{3}\right)$ gas sensing characteristics of pristine GO and functionalized GO, corresponding to the $\mathrm{NH}_{3}$ concentration of 20 to $100 \mathrm{ppm}$ are shown in Fig. 7(a). The percentage response (\% S) and percentage recovery (\% Rec) was calculated using the following equations, ${ }^{32,39}$

$$
S=\frac{Z_{\mathrm{NH}_{3}}-Z_{\text {Air }}}{Z_{\text {Air }}} \times 100 \%
$$

$$
\mathrm{Rec}=\frac{\% S_{\mathrm{NH}_{3}}-\% S_{\mathrm{Air}}}{\% S_{\mathrm{NH}_{3}}} \times 100 \%
$$

where, $Z_{\mathrm{NH}_{3}}$ is impedance of the sensor in ammonia atmosphere and $Z_{\text {Air }}$ is impedance of the Sensor in air.

As from Fig. 7(a) and (b), the sensitivity or response arises due to the interaction between $\mathrm{NH}_{3}$ and $\mathrm{GO}$ based sensor surface (GO and GO-Ar) via physisorption as well as chemisorption. Due to the electrostatic exchange of negative charge carriers from $\mathrm{NH}_{3}$ to the GO sensor surface, the charge carrier density of the sensor has greatly increased, leading to its decrement in electrical impedance $(Z) .{ }^{\mathbf{3 9 , 4 2 , 4 3}}$ During electrostatic exchange of charge carriers, $\mathrm{NH}_{3}$ acts as electron donor (due to its p-type nature) ${ }^{\mathbf{4 1}}$ and functional groups (Ar moieties and oxygen containing groups) on sensor surface acts as electron acceptor. Therefore, we have observed the sensitivity of all sensors in the negative axis. Generally, the surface of GO contains epoxy, hydroxyl and other oxygen containing groups in the intermediate region between $\mathrm{O}$-rich and $\mathrm{H}$-rich surface. ${ }^{40}$ So that, the molecular interaction of $\mathrm{NH}_{3}$ with $\mathrm{GO}$ could be occurred through covalent or non-covalent bonding. For example, the hydrogen bonding between ammonia and hydroxyl groups in GO occurred by the coordination of $\mathrm{N}$ in $\mathrm{NH}_{3}$ and $\mathrm{H}$ in hydroxyl group $(\mathrm{OH} \cdots \mathrm{N})$. Whereas, the hydrogen bonding between ammonia and epoxide groups in GO occurred by the coordination between $\mathrm{H}$ in $\mathrm{NH}_{3}$ and $\mathrm{O}$ in epoxide group $(\mathrm{NH} \cdots \mathrm{O})$. Since, due to its various interactions between ammonia and oxygen containing functional groups on GO, we have observed $9.5 \%$ response under $20 \mathrm{ppm}$ ammonia gas concentration for pristine GO. The sensitivity of pristine GO has increased when the ammonia concentration was increased from 20 to $100 \mathrm{ppm}$.

In order to improve the ammonia sensing properties of GO, it has been functionalized with different derivatives of aryl propargyl ethers (Ar1, Ar2 and Ar3) as shown in Fig. 1. When compared to pristine GO, all functionalized GO sensors exhibited superior ammonia responses and the representative ammonia response curves are shown in Fig. 7(a) and (b). The observed sensitivity are of $28.2 \%$ for GO-Ar1, $63 \%$ for GO-Ar2 and $60 \%$ for GO-Ar3 under $20 \mathrm{ppm}$. The enhanced sensitivity of GO-Ar1 is 3 times higher than the pristine GO, whereas the 

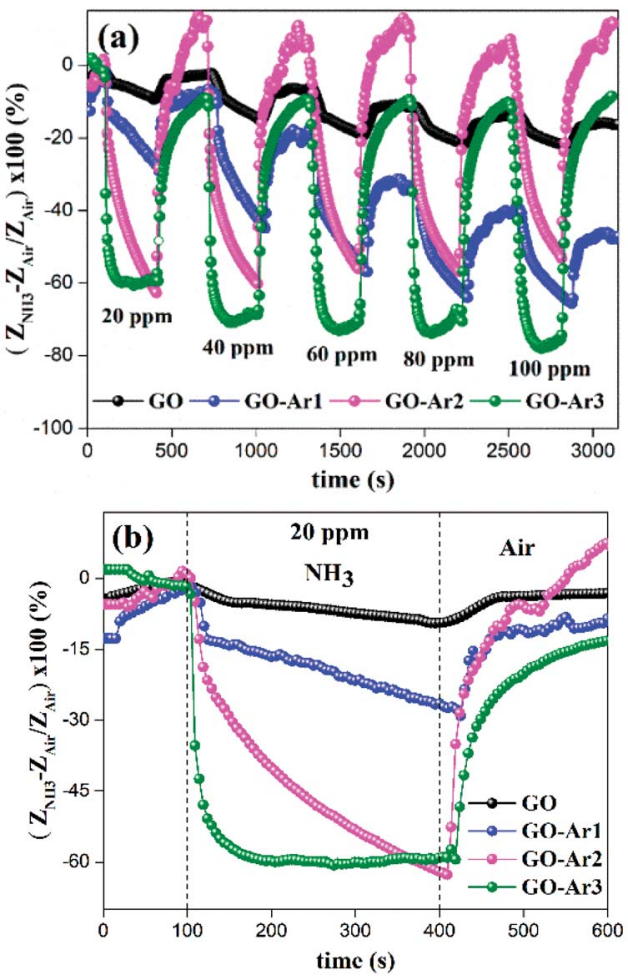

Fig. 7 (a) and (b) Ammonia gas sensing curves of GO and functionalized GO samples.

sensitivity of GO-Ar2 and GO-Ar3 are approximately 6.5 times higher than that of pristine GO. The possible reasons for the enhancement in the sensitivity of functionalized GO are primarily due to the presence of more active sites for adsorption of $\mathrm{NH}_{3}$ molecules on the surface (methyl in Ar1, fluorine in Ar2, $\mathrm{Ar} 3$ and oxygen containing groups on $\mathrm{GO}$ ) and electrostatic interaction with surface functionalities through the functionalization of aryl propargyl ether moieties on GO. Additionally, the vacancies which are produced in hexagonal carbon structure due to the oxidation and further functionalization can also enhances the ammonia adsorption. ${ }^{44}$ In order to verify and support the experimental results, the computational studies were performed on GO and functionalized GO to analyse the interaction between ammonia and GO based sensing material. From the computation results, the calculated adsorption energies of GO, GO-Ar1, GO-Ar2 and GO-Ar3 are $-1.74,-2.14$, $-2.89,-2.63 \mathrm{eV}$ respectively, it reveals that the functionalization of aryl propargyl ether on GO leads to the high adsorption of ammonia gas molecules. When compared to the recovery of pristine GO (72\%), all functionalized GO sensors exhibit high percentage recoveries $(74 \%$ for GO-Ar1, $100 \%$ for GO-Ar2 and 91\% for GO-Ar3) at room temperature. The summarized results are given in Table 1, it was observed that the functionalized GO sensor displays swift response and recovery than that of pristine GO.

The high electronegative aryl propargyl ether functionalized GO sensors (GO-Ar2 and GO-Ar3) exhibits superior gas response and recovery than other $\mathrm{GO}$ functionalized sensing material due to the substitutions of fluorine atom, which has greatly enhanced the ammonia binding towards the surface GO-Ar2 and GO-Ar3. As observed from Fig. 7(a) and (b), the GO-Ar3 sensor exhibits sensitivity $(60 \%)$ with swift response time of 78 seconds due to tri-fluorine substitution, where as a gradual increase in the sensitivity with longer response time $(305$ seconds) has been observed for GO-Ar2 due to di-fluorine substitution. It has been observed that the percentage recovery was reduced when increasing the fluorine substitution on GO-Ar, might be due to the strong chemisorption. The calculated response and recovery time of GO and functionalized GO are summarized in the Table 1.

Reports suggest that for metal oxide based gas sensors, due to strong chemisorption of gas species, external heating is required to overcome the activation barrier to achieve complete recovery. ${ }^{45,46}$ However, heating requires additional electrical circuits, which will increase the complexity of sensor device fabrication. However, in this work, we have observed a nearly complete recovery (more than 90\%) in GO-Ar2 and GO-Ar3 sensor device, without any external heating. When compared to previous reported results (given in Table 2), the di-fluoro and tri-fluoro substituted aryl propargyl ether functionalized GO exhibits higher $\mathrm{NH}_{3}$ gas response and recovery at room temperature.

\subsection{Humidity analysis}

To explore the effect of relative humidity (RH) on ammonia gas response of the pristine $\mathrm{GO}$ and functionalized GO (GO-Ar2 and GO-Ar3), the sensors were exposed to different relative humidity environments (different humidity levels were maintained inside the chamber by using saturated saline solutions). After achieving constant humidity inside the chamber, ammonia $(20$ $\mathrm{ppm})$ was allowed to enter into the chamber and the response was determined under different humid conditions. Fig. 8(a-d) shows the representative ammonia response curve of pristine GO, GO-Ar1, GO-Ar2 and GO-Ar3 under different humidity levels such as $11 \%, 51 \%$ and $97 \%$, respectively. It has been observed from Fig. $8(\mathrm{e})$ that, there is a significant enhancement in the

Table 1 The pristine $\mathrm{GO}$ and functionalized GO gas sensing curve properties

\begin{tabular}{lllll}
\hline Sensing material & $\begin{array}{l}\text { Response time } \\
\text { at } 20 \mathrm{ppm}(\mathrm{s})\end{array}$ & $\begin{array}{l}\text { Recovery time } \\
\text { at } 20 \mathrm{ppm}(\mathrm{s})\end{array}$ & $\begin{array}{l}\text { Max. \% } \\
\text { response at 20 ppm }\end{array}$ & $\begin{array}{l}\text { Max. \% } \\
\text { recovery at 20 ppm }\end{array}$ \\
\hline GO & 314 & 287 & 9.5 & 72 \\
GO-Ar1 & 312 & 90 & 28.2 & 74 \\
GO-Ar2 & 305 & 220 & 63 & 100 \\
GO-Ar3 & 78 & 260 & 60 & 91
\end{tabular}


Table 2 Comparative study with previous works reported by other researchers

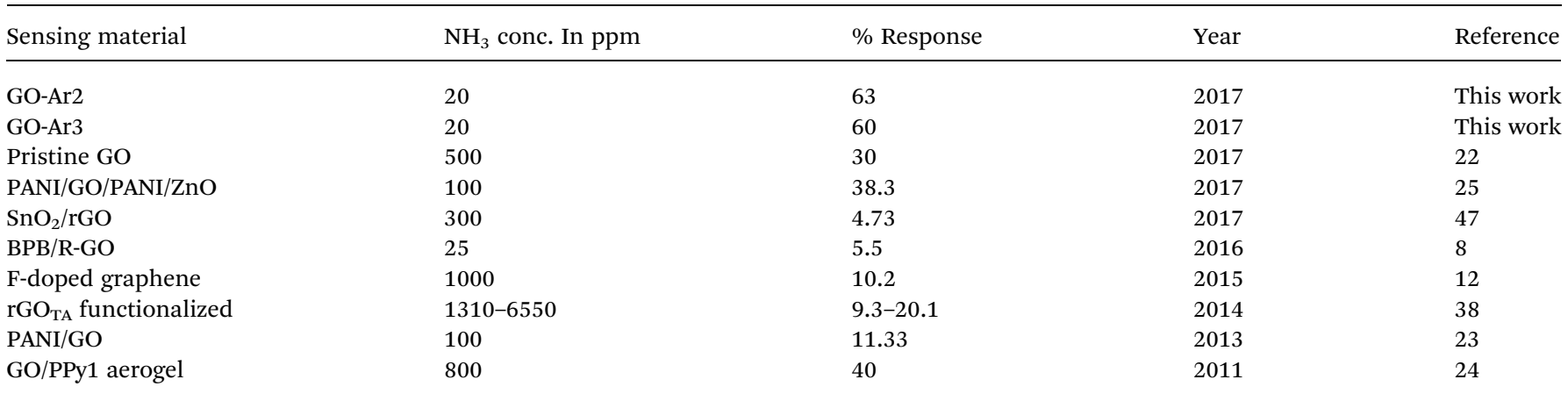

ammonia response from 15 to $34 \%$ for pristine GO, 20 to $45 \%$ for GO-Ar1, 51 to $81 \%$ for GO-Ar2 and 58.2 to $77.6 \%$ for GO-Ar3 by increasing the humidity level from $11 \%$ to $97 \%$. This can be attributed to the presence of Ar moieties and oxygen containing functional groups on the sensing surface, which forms a molecularly thin water layer on the surface. The thickness of
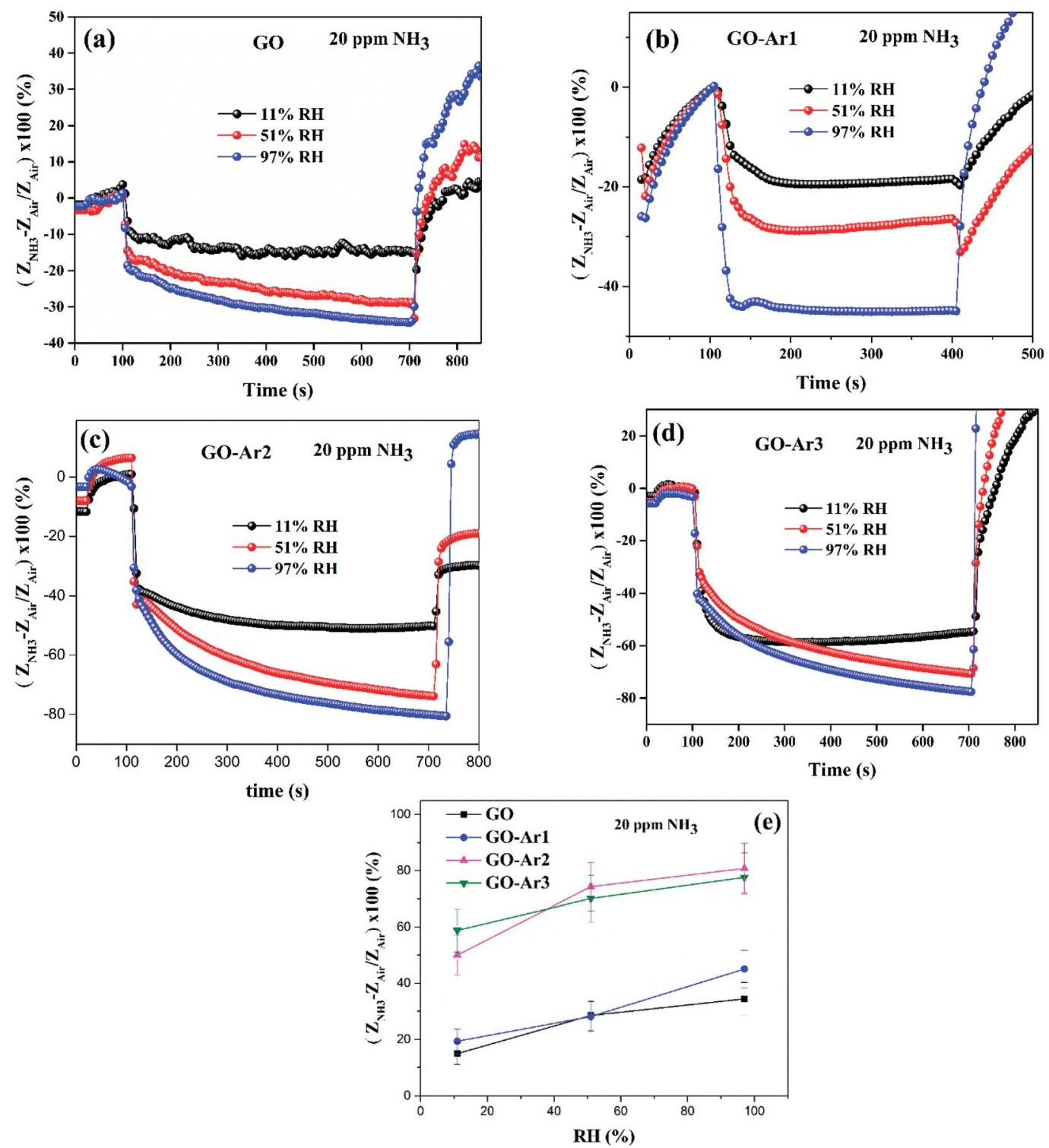

Fig. 8 (a), (b) and (c) Ammonia gas sensing curves of GO, GO-Ar2 and GO-Ar3 under different humidity environments, (d) \% sensitivity vs. \% RH plot. 
surface water layer increases with increase in $\mathrm{RH} .{ }^{48}$ The presence of water layer on the sensor surface can enhance the adsorption of ammonia gas molecule, as reported by Lechner et al. ${ }^{49}$ The following acid-base reaction of $\mathrm{NH}_{3}$ with $\mathrm{H}_{2} \mathrm{O}$ forms aqueous ammonia with protonated $\mathrm{NH}_{4}{ }^{+}$:

$$
\mathrm{NH}_{3}+\mathrm{H}_{2} \mathrm{O}=\mathrm{NH}_{4}^{+}+\mathrm{OH}^{-}
$$

Which leads to the electron transfer from hydrated ammonia to sensing material, thereby decreasing the impedance and hence increasing ammonia sensitivity.

The GO-Ar1 displays 1.28, 0.9,1.32 and GO-Ar2 shows 3.4, 2.64, 2.38 times higher $\mathrm{NH}_{3}$ response than pristine GO under the $\mathrm{RH}$ conditions of $11 \%, 51 \%$ and $97 \%$, respectively. Whereas, GO-Ar3 exhibits 3.88, 2.5 and 2.28 times higher $\mathrm{NH}_{3}$ response than pristine GO under the $\mathrm{RH}$ conditions of $11 \%, 51 \%$ and $97 \%$, respectively. This enhanced ammonia response of GO-Ar2 and GO-Ar3 attributed to the high interaction potential towards ammonia (due to the functionalization of fluorine substituted aryl propargyl ether) and more hydrophilic nature (due to surface fluorine atoms) than GO.

\subsection{Gas selectivity analysis}

We have carried out the gas sensitivity measurement of GO-Ar3 under different gas environments (20 ppm) such as ammonia, acetone, ethanol and dichloromethane (DCM), nitrogen
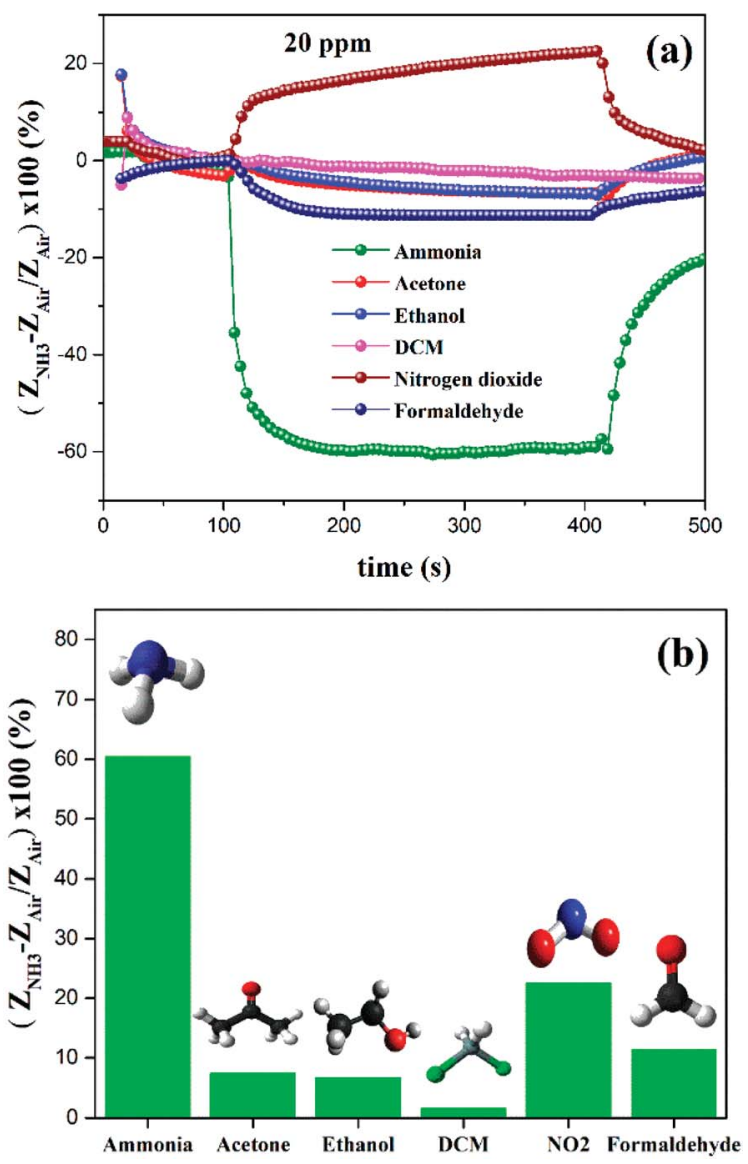

Fig. 9 (a) Gas sensitivity curves of GO-Ar3 and (b) gas selectivity plot. dioxide, formaldehyde respectively. The corresponding sensitivity curves of GO-Ar3 under different gas environments are shown in Fig. 9(a) and (b). It has been observed that among all the investigated gasses, GO-Ar3 shows significantly higher response towards ammonia. The sensitivity of GO-Ar3 determined under $20 \mathrm{ppm}$ of ammonia, acetone, ethanol, DCM, nitrogen dioxide and formaldehyde are $60 \%, 7.4 \%, 6.7 \%$, $1.54 \%, 22.5 \%, 11.2 \%$ respectively. Hence, the functionalization of GO with Ar3 makes superior selectivity towards ammonia sensing application.

\section{Computation}

\subsection{Computational methods}

Density functional theory calculation via grid-based projector augmented wave (GPAW) method is implemented. ${ }^{50}$ Exchange correlation of the Perdew-Burke-Ernzerhof (PBE) exchange correlation with spin polarization are applied for all calculations. ${ }^{51}$ All calculations are carried out in $\Gamma$ point where $15 \AA$ of vacuum is applied in all direction.

$\mathrm{NH}_{3}$ adsorption energy over functionalized graphene is calculated by the eqn (4):

$$
\left.E_{\mathrm{b}}=E\left[\mathrm{GO}(\operatorname{Arx})+\mathrm{NH}_{3}\right]-E[\mathrm{GO}(\operatorname{Arx})]-E\left[\mathrm{NH}_{3}\right]\right)
$$

Negative energy indicates exothermic reaction.

\subsection{Computational results}

$\mathrm{NH}_{3}$ adsorption over functionalized graphene oxides is performed within density functional theory. $\mathrm{NH}_{3}$ is adsorbed over GO, GO-Ar1, GO-Ar2, and GO-Ar3. The atomic model and adsorption energy of $\mathrm{NH}_{3}$ over Go, GO-Ar1, GO-Ar2, and GO-Ar3 are shown and collected in Fig. 10 and Table 3. Please note that $\mathrm{H}$ atom in GO-Ar2 is diffused from GO to $\mathrm{C}_{6} \mathrm{~F}_{2}$ as shown in Fig. 10(c) upon the relaxation. $\mathrm{NH}_{3}$ is adsorbed at the edge of $\mathrm{O}$
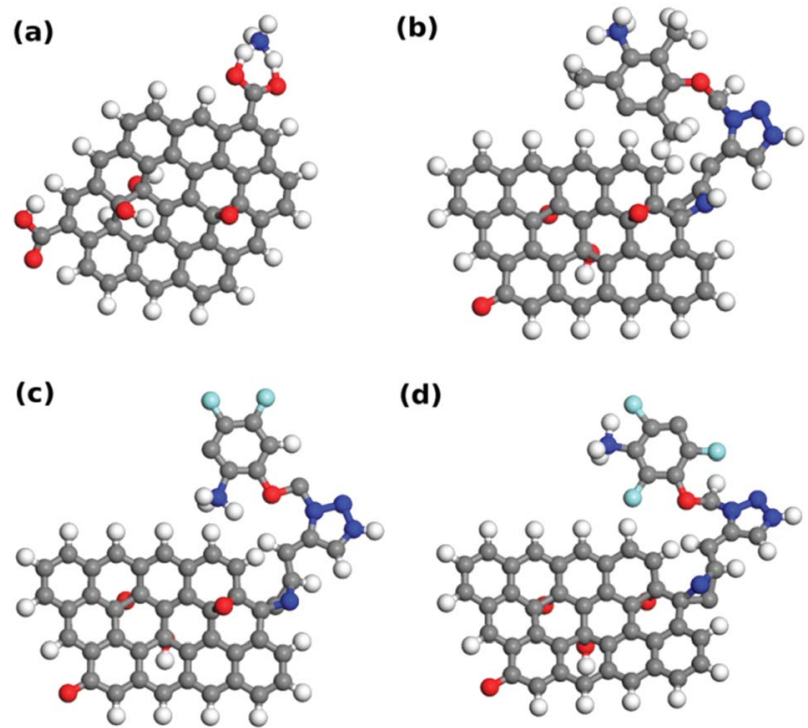

Fig. 10 Atomic model of (a) GO, (b) GO-Ar1, (c) GO-Ar2, and (d) GOAr3. Atomic color code: C; gray, $\mathrm{H}$; white, O; red, N; blue, F; light blue. 
Table 3 Adsorption energy (E) in eV of NH3 over Go, GO-Ar1, GO-Ar2, and GO-Ar3

\begin{tabular}{ll}
\hline Samples & $E(\mathrm{eV})$ \\
\hline GO & -1.74 \\
GO-Ar1 & -2.14 \\
GO-Ar2 & -2.89 \\
GO-Ar3 & -2.63
\end{tabular}

atoms in $\mathrm{GO}$ while $\mathrm{NH}_{3}$ is adsorbed at $\mathrm{C}$ atom of functionalized $\mathrm{C}_{6}$ as shown in Fig. 10. Table 3 shows that the adsorption energy of $\mathrm{NH}_{3}$ over GO, GO-Ar1, GO-Ar2, and GO-Ar3 is calculated to be $-1.71 \mathrm{eV},-2.14 \mathrm{eV},-2.89 \mathrm{eV}$, and $-2.63 \mathrm{eV}$, respectively. One can see that $\mathrm{NH}_{3}$ adsorption energy is enhanced with functionalized graphene oxide (Ar1, Ar2, and Ar3) compared to $\mathrm{NH}_{3}$ over GO. In particular, high $\mathrm{NH}_{3}$ adsorption energy is observed with the addition of $\mathrm{F}$ atoms as seen in GO-Ar2 and GO-Ar3. This implies that $\mathrm{F}$ atoms are considered to be a key element for enhancing the sensitivity of GO against $\mathrm{NH}_{3}$.

The electronic structure of $\mathrm{NH}_{3}$ over GO, GO-Ar1, GO-Ar2, and GO-Ar3 are investigated in order to reveal the enhancement of $\mathrm{NH}_{3}$ adsorption upon the introduction of $\mathrm{F}$ atoms. In particular, projected density of states (PDOS) of $\mathrm{NH}_{3}$ adsorbed over GO-Ar1 and GO-Ar3 are calculated and shown in Fig. 11. Fig. 11(a) shows that overlapping of p-electrons of $\mathrm{C}$ in $\mathrm{CH}_{3}$ and p-electrons of $\mathrm{N}$ is weak while strong overlapping is seen of $\mathrm{p}$ electrons of $\mathrm{F}$ and p-electrons of $\mathrm{N}$ in Fig. 11(b). Thus, Fig. 11
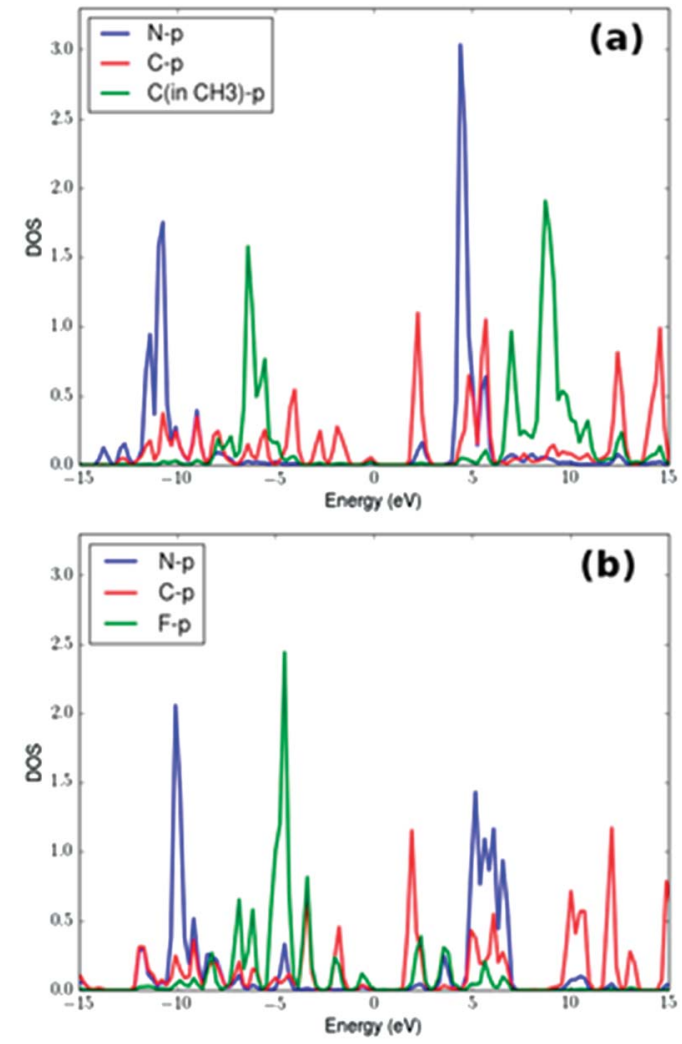

Fig. 11 Projected density of states (PDOS) of NH3 adsorbed (a) GOAr1 and (b) GO-Ar3. demonstrates that the p-electrons of $\mathrm{F}$ atoms are contributing to the adsorption of $\mathrm{NH}_{3}$, therefore enhancement of $\mathrm{NH}_{3}$ adsorption energy is induced.

\section{Conclusions}

This work reports the successful functionalization of GO with terminal azide $\left(\mathrm{N}_{3}\right)$ of GO and aryl propargyl ether derivatives (Ar1, Ar2 and Ar3) by using Cu-catalyzed Huisgen 1,3-dipolar cycloaddition reaction. The structural, microstructural, spectroscopic, thermal, and surface properties of the samples were investigated. Furthermore, we have analysed the ammonia sensitivity of aryl propargyl ether derivatives functionalized GO. Compared to pristine GO, aryl propargyl ether functionalized GO shows excellent $\mathrm{NH}_{3}$ sensitivity. The enhanced ammonia sensitivity of functionalized GO samples were supported through computational studies. Due to high adsorption energy $(-2.89 \mathrm{eV}$ and $-2.63 \mathrm{eV})$, GO-Ar2 and GO-Ar3 exhibit a superior ammonia response of $63 \%$ and $60 \%$, respectively, at $20 \mathrm{ppm}$ which is approximately 6.4 times higher than that of pristine GO (-9.5\%). Also nearly full sensor recovery (more than 90\%) was achieved without any heat treatment. Additionally, the $\mathrm{NH}_{3}$ sensing response of GO-Ar2 and GO-Ar3 (at 20 ppm) was found to increase with the increase of relative humidity values ranging from $11 \%$ to $97 \%$. Among the investigated different gases, GOAr3 shows high gas selectivity towards ammonia. Compared to pristine GO, these aryl propargyl ether functionalized graphene oxide materials can be useful for ammonia gas sensing applications.

\section{Conflicts of interest}

Author has no conflicts of interest.

\section{Acknowledgements}

The author Farheen Khurshid thankfully acknowledge UGC, New Delhi for MNAF research fellowship. MSLH is thankful to Department of Science and Technology, New Delhi for INSPIRE Faculty grant (IFA12-PH-18). The necessary experimental facilities provided by the Central University of Tamil Nadu (CUTN), Thiruvarur, and Karunya University, Coimbatore are gratefully acknowledged. This work is also funded by JSPS KAKENHI, Grant-in-Aid for Young Scientists (B), Grant Number JP17K14803 and "Materials research by Information Integration" Initiative ( $\left.\mathrm{MI}^{2} \mathrm{I}\right)$ project of the Support Program for Starting Up Innovation Hub from Japan Science and Technology Agency (JST) and Computational work is supported in part by Hokkaido university academic cloud information initiative centre, Hokkaido University, Sapporo, Japan.

\section{References}

1 D. Zhang, C. Jiang, P. Li and Y. e. Sun, ACS Appl. Mater. Interfaces, 2017, 9, 6462-6471.

2 D. Ponnusamy and S. Madanagurusamy, J. Electron. Mater., 2015, 44, 4726. 
3 Y. Jia, C. Yan, H. Yu, L. Chen and F. Dong, Sens. Actuators, B, 2014, 203, 459-464.

4 S. Abdulla, T. L. Mathew and B. Pullithadathil, Sens. Actuators, B, 2015, 221, 1523-1534.

5 C.-C. Cheng, C.-L. Wu, Y.-M. Liao and Y.-F. Chen, ACS Appl. Mater. Interfaces, 2016, 8, 17382-17388.

6 M. Pumera, A. Ambrosi, A. Bonanni, E. L. K. Chng and H. L. Poh, TrAC, Trends Anal. Chem., 2010, 29, 954-965.

7 S. Tang and Z. Cao, J. Phys. Chem. C, 2012, 116, 8778-8791.

8 H. Song, X. Li, P. Cui, S. Guo, W. Liu and X. Wang, Diamond Relat. Mater., 2017, 73, 56-61.

9 F. Schedin, A. Geim, S. Morozov, E. Hill, P. Blake, M. Katsnelson and K. Novoselov, Nat. Mat., 2007, 6, 652-655.

10 L. T. Duy, T. Q. Trung, V. Q. Dang, B. U. Hwang, S. Siddiqui, I. Y. Son, S. K. Yoon, D. J. Chung and N. E. Lee, Adv. Funct. Mater., 2016, 26, 4329-4338.

11 X. Huang, N. Hu, R. Gao, Y. Yu, Y. Wang, Z. Yang, E. S.-W. Kong, H. Wei and Y. Zhang, J. Mater. Chem., 2012, 22, 22488-22495.

12 S. Prezioso, F. Perrozzi, L. Giancaterini, C. Cantalini, E. Treossi, V. Palermo, M. Nardone, S. Santucci and L. Ottaviano, J. Phys. Chem. C, 2013, 117, 10683-10690.

13 M. Katkov, V. Sysoev, A. Gusel'Nikov, I. Asanov, L. Bulusheva and A. Okotrub, Phys. Chem. Chem. Phys., 2015, 17, 444-450.

14 R. Lv, G. Chen, Q. Li, A. McCreary, A. Botello-Méndez, S. Morozov, L. Liang, X. Declerck, N. Perea-López and D. A. Cullen, Proc. Natl. Acad. Sci.,, 2015, 112, 14527-14532.

15 A. G. Bannov, J. Prášek, O. Jašek, A. A. Shibaev and L. Zajíčková, Procedia Eng., 2016, 168, 231-234.

16 S. K. Hazra and S. Basu, C, 2016, 2, 12.

17 K. Toda, R. Furue and S. Hayami, Anal. Chim. Acta, 2015, 878, 43-53.

18 K. I. Han, S. Kim, I. G. Lee, J. P. Kim, J.-H. Kim, S. W. Hong, B. J. Cho and W. S. Hwang, Sensors, 2017, 17, 407.

19 N. L. Teradal, S. Marx, A. Morag and R. Jelinek, J. Mater. Chem. C, 2017, 5, 1128-1135.

20 Z. Xu, K. Wu, S. Zhang, Y. Meng, H. Li and L. Li, Mater. Horiz., 2017, 4, 383-388.

21 A. Pazat, E. Beyou, C. Barrès, F. Bruno and C. Janin, Appl. Surf. Sci., 2017, 396, 902-911.

22 W. S. Hummers Jr and R. E. Offeman, J. Am. Chem. Soc., 1958, 80, 1339.

23 A. G. Bannov, J. Prášek, O. Jašek and L. Zajíčková, Sensors, $2017,17,320$.

24 Z. Wu, X. Chen, S. Zhu, Z. Zhou, Y. Yao, W. Quan and B. Liu, Sens. Actuators, B, 2013, 178, 485-493.

25 H. Bai, K. Sheng, P. Zhang, C. Li and G. Shi, J. Mater. Chem., 2011, 21, 18653-18658.

26 D. Zhang, Z. Wu, P. Li, X. Zong, G. Dong and Y. Zhang, Sens. Actuators, B, 2018, 258, 895-905.

27 D. Zhang, C. Jiang and Y. e. Sun, J. Alloys Compd., 2017, 698, 476-483.
28 M. Jeyavelan, A. Ramesh, R. R. Kannan, T. Sonia, K. Rugunandhiri and M. S. L. Hudson, RSC Adv., 2017, 7, 31272-31280.

29 L. Kou, H. He and C. Gao, Nano-Micro Lett., 2010, 2, 177-183.

30 D. Zhang, A. Liu, H. Chang and B. Xia, $R S C A d v ., 2015,5$, 3016-3022.

31 X. Wang, F. Cui, J. Lin, B. Ding, J. Yu and S. S. Al-Deyab, Sens. Actuators, B, 2012, 171, 658-665.

32 B. K. Mutuma, R. Rodrigues, K. Ranganathan, B. Matsoso, D. Wamwangi, I. A. Hümmelgen and N. J. Coville, J. Mater. Chem. A, 2017, 5, 2539-2549.

33 D. Evrard, F. Lambert, C. Policar, V. Balland and B. Limoges, Chem. -Eur. J., 2008, 14, 9286-9291.

34 K. N. Kudin, B. Ozbas, H. C. Schniepp, R. K. Prud'Homme, I. A. Aksay and R. Car, Nano Lett., 2008, 8, 36-41.

35 D. Egloff, I. A. Oleinich, M. Zhao, S. L. König, R. K. Sigel and E. Freisinger, ACS Chem. Biol., 2016, 11, 2558-2567.

36 M. S. L. Hudson, K. Takahashi, A. Ramesh, S. Awasthi, A. K. Ghosh, P. Ravindran and O. N. Srivastava, Catal. Sci. Technol., 2016, 6, 261-268.

37 T. Zhou, F. Chen, K. Liu, H. Deng, Q. Zhang, J. Feng and Q. Fu, Nanotechnology, 2010, 22, 045704.

38 Y. Chen, S. A. Freunberger, Z. Peng, F. Bardé and P. G. Bruce, J. Am. Chem. Soc., 2012, 134, 7952-7957.

39 Q. Lin, Y. Li and M. Yang, Sens. Actuators, B, 2012, 173, 139147.

40 W. Cai, R. D. Piner, F. J. Stadermann, S. Park, M. A. Shaibat, Y. Ishii, D. Yang, A. Velamakanni, S. J. An and M. Stoller, Science, 2008, 321, 1815-1817.

41 H. Chang, J. D. Lee, S. M. Lee and Y. H. Lee, Appl. Phys. Lett., 2001, 79, 3863-3865.

42 B. Kumar, K. Min, M. Bashirzadeh, A. B. Farimani, M.-H. Bae, D. Estrada, Y. Kim, P. Yasaei, Y. Park and E. Pop, Nano Lett., 2013, 13, 1962-1968.

43 G. Lu, L. E. Ocola and J. Chen, Appl. Phys. Lett., 2009, 94, 083111.

44 R. Ghosh, A. Midya, S. Santra, S. K. Ray and P. K. Guha, ACS Appl. Mater. Interfaces, 2013, 5, 7599-7603.

45 C. S. Rout, M. Hegde, A. Govindaraj and C. Rao, Nanotechnology, 2007, 18, 205504.

46 E. Llobet, G. Molas, P. Molinas, J. Calderer, X. Vilanova, J. Brezmes, J. Sueiras and X. Correig, J. Electrochem. Soc., 2000, 147, 776-779.

47 D. Zhang, J. Liu, C. Jiang, A. Liu and B. Xia, Sens. Actuators, B, 2017, 240, 55-65.

48 D. B. Asay and S. H. Kim, J. Phys. Chem. B, 2005, 109, 1676016763.

49 B. A. Lechner, Y. Kim, P. J. Feibelman, G. Henkelman, H. Kang and M. Salmeron, J. Phys. Chem. C, 2015, 119, 23052-23058.

50 J. J. Mortensen, L. B. Hansen and K. W. Jacobsen, Phys. Rev. B: Condens. Matter Mater. Phys., 2005, 71, 035109.

51 J. P. Perdew, K. Burke and M. Ernzerhof, Phys. Rev. Lett., 1996, 77, 3865. 